\title{
A Semi-Canonical Form for Sequential AIGs
}

\author{
Alan Mishchenko Niklas Een Robert Brayton \\ Department of EECS, University of California, Berkeley \\ \{alanmi, brayton\}@eecs.berkeley.edu niklas@een.se
}

\begin{abstract}
In numerous EDA flows, time-consuming computations are repeatedly applied to sequential circuits. This motivates developing methods to determine what circuits have been processed already by a tool. This paper proposes an algorithm for semi-canonical labeling of nodes in a sequential AIG, allowing problems or sub-problems solved by an EDA tool to be cached with their computed results. This can speed up the tool when applied to designs with isomorphic components or design suites exhibiting substantial structural similarity.
\end{abstract}

\section{Introduction}

In sequential and-inverter-graphs (AIGs) there frequently exist many instances of the same logic sub-circuit but with different inputs. Many synthesis and verification packages spend significant time analyzing logic, and the presence of identical sub-circuits results in duplication of effort.

Alternatively, if a synthesis or verification package is invoked on a design and then again on a minimally changed version of the same design, many of the logic sub-circuits remain unchanged between runs. Time spent re-analyzing these sub-circuits results in a duplication of effort.

The method proposed in this paper for semi-canonical labeling of sequential AIGs, detects the majority of isomorphic sub-circuits. Intermediate results computed on these sub-circuits can be cached and applied across every instance of the sub-circuit, leading to dramatic improvements in runtimes of synthesis or verification.

A sequential AIG represents a sequential circuit as a directed labeled graph consisting of two-input AND nodes, primary inputs, primary outputs, and flip-flop nodes. The edges are labeled with 1 denoting inversion or 0 otherwise. Labeling of nodes and AND gate fanins can be arbitrary.

The idea of the method is to use this freedom, to re-label the nodes based on their transitive fanin and fanout graph structures, and thereby create a semi-canonical form for the given sequential AIG. Thus a new labeling is derived and each node's fanins are listed in numerical order of their labels. We want the semi-canonical structure to be as precise as reasonably possible. For example, given two isomorphic AIGs, we want their newly labeled representations to be identical. If this always happens, the labeling is canonical. While it is not efficient to make relabeling exactly precise in this sense, we want it to be highly precise in that it rarely fails to identify two isomorphic AIGs. We call this labeling semi-canonical.
Michael Case Pankaj Chauhan Nikhil Sharma

Calypto Design Systems

\{mcase, pchauhan, nsharma\}@calypto.com

Three applications of this idea are as follows.

1. It can detect structurally isomorphic primary outputs (POs) of a multi-output sequential AIG. This is done by taking each output's cone and mapping it into its semi-canonical form. If the forms of the two outputs, are identical, then the outputs are isomorphic.

2. In verification, where each output represents a property to be proved, if two outputs are isomorphic then it is only necessary to solve one. If an inductive invariant (or a counter-example) is computed to witness a proof (or a failure) of one property, it can be readily remapped to be a witness for the other. Thus when verifying a set of outputs, only one representative of each isomorphic class need be considered, reducing the number of proof obligations.

3. It can be used to cache synthesis or verification results that have been computed previously on an AIG. When an AIG is to be processed, it is cast into its semi-canonical form and stored in a cache along with the computed result. Given a new AIG, we compute its semi-canonical form and check if it is cached. If already cached, we return the saved result. Otherwise, we solve the problem and cache the semicanonical form and the result.

The method finds a re-labeling of the node IDs using the AIG structure. This is done by computing signatures for each node, based the node's transitive fanin cone in the sequential AIG. Unique labels are assigned to a node that has a unique signature. For nodes with non-unique signatures, "tie-breaking" is done by assigning one such node an unused label and then node signatures are updated based on this. The key for this semi-canonicalization is a) to define the signature of a node in such a way that it is very precise, and b) to compute and update signatures efficiently.

To summarize, the contributions of this paper are:

- An algorithm for structural semi-canonical labeling for sequential AIGs is given. The method of re-labeling can be adapted easily to other forms of graphs.

- Analysis of public and industrial benchmarks confirms that a) re-labeling can be computed efficiently, and b) many benchmarks contain a large number of isomorphic primary output cones.

The paper is organized as follows. Section 2 describes some background. Section 3 describes the proposed algorithms for semi-canonical labeling. Section 4 reports experimental results and Section 5 concludes and outlines future work. 


\section{Background}

A sequential $A I G$ has a constant node, primary inputs and outputs, flop inputs and outputs, and internal AND nodes. It has node attributes $\{P I, P O, F F$, internal $\}$ and the edge attributes $\{$ direction, complementation $\}$. Two graphs are identical $(H \equiv G)$ if their representations are identical. Checking this can be done by writing the two graphs into files and verifying their contents to be identical (using diff).

The conventional representation of an AIG [2] is a sequence of triplets $\left\{\left(a_{1}, b_{1}, c_{1}\right), \ldots,\left(a_{N}, b_{N}, c_{N}\right)\right\}$ where $a_{i}, b_{i}, c_{i}$ are node labels, $a_{i}$ identifying a 2-input AND gate and $b_{i}, c_{i}$ its two fanin nodes. $b_{i}, c_{i}$ are negated node labels if the corresponding edge contains an invertor. The parent node $\left\{a_{i}\right\}$, must appear in topological order in the list, and each child pair $\left(b_{i}, c_{i}\right)$ must be in ascending order.

An isomorphism $f$ between two attributed graphs, $H$ and $G$, is a 1-1 mapping $f: \mathrm{V}(G) \rightarrow \mathrm{V}(H)$, where nodes $(u, f(u))$ have the same attributes, and edges $(u \rightarrow v, f(u) \rightarrow f(v))$ have the same attributes. Two graphs are isomorphic ( $G \approx H$ ) if an isomorphism exists between the two. Given $G, H \approx G$ can be derived by relabeling its set of nodes $\mathrm{V}(G)$ randomly.

Graph canonization is the problem of finding a mapping canon: $\mathrm{V}(G) \rightarrow \mathrm{V}(G)$ such that

1. $\operatorname{canon}(G) \approx G$, and

2. $[\operatorname{canon}(H) \equiv \operatorname{canon}(G)] \Leftrightarrow[H \approx G]$.

$\operatorname{canon}(\mathrm{G})$ is said to be a canonical form for $\mathrm{G}$ and canon is said to be a canonical mapping for $\mathrm{G}$.

We give an algorithm for re-labeling of nodes of $\mathrm{V}(G)$ ), iso, such that $i s o(G) \approx G$. Clearly if $i s o(H) \equiv i s o(G)$, then $H \approx G$. We do not claim that iso is a canonical mapping, but we do claim that for the majority of graphs if $H \approx G$, then $i \operatorname{so}(H) \equiv i \operatorname{so}(G)$. We call such a mapping a semicanonical mapping.

Although canonical labeling is well-studied [14], this paper considers the special case of sequential AIG graphs.

\section{Algorithm}

\subsection{Motivating example}

Consider the AIG with inputs $\{a, b, c, d\}$ and outputs $\{F, G\}$, shown on the left of Figure 3.1. A dotted edge denotes inversion.

To compute a semi-canonical labeling of the objects in the AIG, we need to distinguish the nodes using their graph properties, independent of their current names.

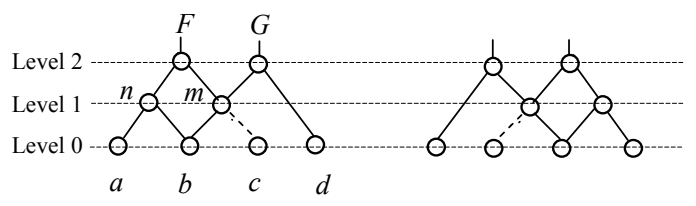

Figure 3.1: Illustration of canonical labeling.
For example, the above circuit has one PI $(b)$ and one internal node $(m)$ with two fanouts. Since these nodes are unique on their levels (level 0 and level 1), they differ from other objects based on their level and fanout count. Next, note that there is only one PI (c) pointed to by a complemented edge, which distinguishes it from other PIs. One of the PO nodes $(F)$ has both fanins on the same level, while the other PO node $(G)$ has fanins on different levels. These observations allow the POs to be distinguished. Other nodes $(a, d, n)$ can be distinguished similarly.

At the time when a node is distinguished, it can be assigned a unique label. As a result, if the graphs are isomorphic, their semi-canonical labels will be identical with high probability. If they are, then graph isomorphism is easily checked. The check may fail, but in practice, this seldom happens, using the method proposed in this paper for re-labeling, as experiments attest.

For example, consider the AIG shown on the right of Figure 3.1. If we identify its objects using the same rules as we used for the circuit on the left, we will assign semicanonical labels in the same order. Thus isomorphism is easily established by checking the resulting representations to be identical (one is the mirror image of the other).

\subsection{Computing semi-canonical labels}

This section describes the proposed method for computing a re-labeling of the AIG objects to derive a semi-canonical AIG structure. Each node will be given a signature and a label (or new node id). Each edge will be given a value. We begin by defining an edge value which will depend on the edge source node (driver node) label, whether it is complemented or not, and its driver level. Next, we show how node signatures are computed from neighboring node signatures and the edge values. Then an iterative procedure is given to increase the uniqueness of the signatures and to assign labels to nodes as they are uniquely identified by their signatures. Finally, we describe a tie-breaking method that is invoked when two nodes are not distinguished by their signatures. If tie-breaking is never invoked then the method is precise.

\subsubsection{Computing edge values}

An edge value captures structural properties of an AIG edge, in particular: (a) the presence of the complemented attribute, (b) the logic level of its source node or sink node, and (c) the fact that source or sink node of the edge are assigned a semi-canonical label. Thus, the edge value depends on node label assignments through this computation.

The edge value is computed using procedure getEdgeValue() in Figure 3.2. This procedure takes an edge and the number of the refinement iteration. For the initial iteration (iter $=0$ ), the edge value is computed using two parameters: the level of the source node of the fanin edge, and the complemented attribute of this edge. In other iterations, the level information is replaced by a node label 
if one has been assigned to the driver node. Otherwise, the edge value is 0 .

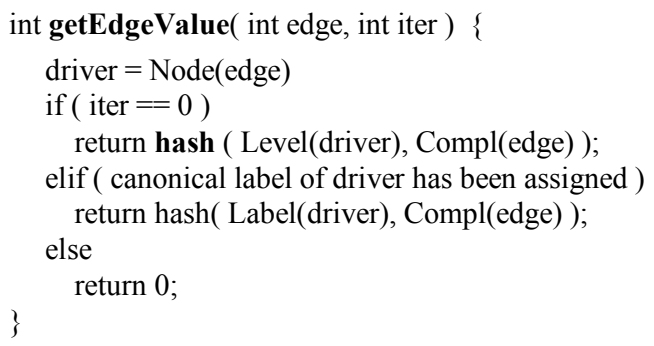

Figure 3.2: Computing edge values.

The first if-statement increases efficiency by increasing the amount of information stored in edge values at the beginning of the computation. The choice of hash function is arbitrary. Using a hash function mapping an interger value into a range of 256 random 32-bit values results in excellent distinguishing power. Function Node() returns the source node of the given incoming edge; $\operatorname{Compl}()$ returns 1 if the edge is complemented and 0 otherwise; Level() returns the logic level of a node in the sequential AIG.

We emphasize that when a node has been assigned a label, subsequent edge values will be affected.

\subsubsection{Node signature propagation}

Initially, all node signatures are set to 0. During signature propagation, the AIG is repeatedly traversed in the forward (backward) direction while edge values are added to node signatures. As a result, some nodes (singleton nodes) acquire unique signatures.

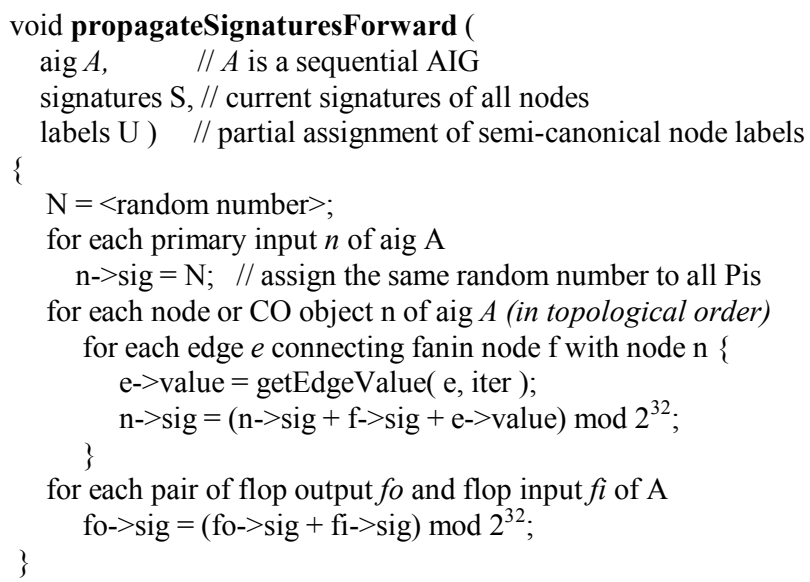

\section{Figure 3.3: Propagating node signatures forward.}

At the end of each forward (backward) traversal, the newly derived singleton nodes are sorted by their signature and assigned labels in that order. These labels impact subsequent edge value computations, as shown in Figure 3.2, and hence subsequent node signature computations. As a result, when a node is visited its updated signature reflects the node labels determined in all previous rounds.

The procedure for propagating node signatures forward is shown in Figure 3.3. It begins by assigning the same random number to all PIs. Next, it considers all internal nodes and COs in a topological order. For each node or $\mathrm{CO}$, it computes edge values for their fanin edges. Next, the node signature is computed as the sum of (a) its old signature, (b) its node-fanin signatures, and (c) its faninedge values. Finally, each flop output node-signature is updated by adding the node signature of its flop input.

The procedure for propagating signatures backward is similar to that shown in Figure 3.3. The differences are:

- starting signatures for primary inputs are not assigned and signatures of the POs are not changed;

- the AIG is traversed in the reverse topological order;

- instead of fanins and fanin edges, fanouts and fanout edges are considered;

- signatures are transferred from flop outputs to flop inputs and not vice versa.

\subsection{Refining node signatures}

We require each node in the sequential AIG to have a unique signature. Refinement refers to the process of updating signatures for every node to reduce the number of nodes having the same signature.

Node signatures for a sequential AIG are computed using signature propagation, as shown in Figure 3.4.

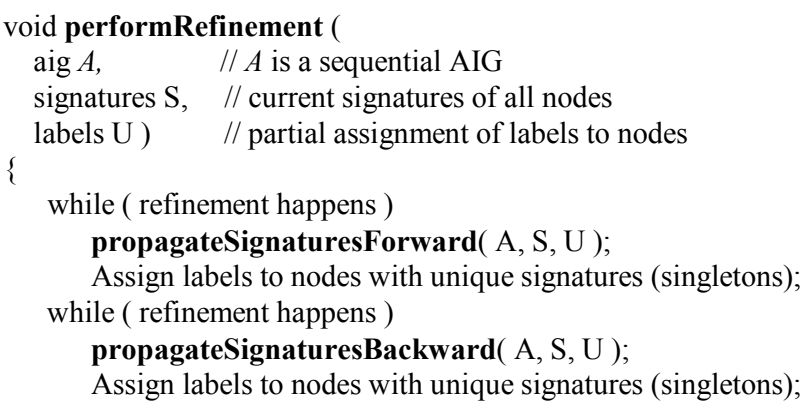

Figure 3.4: Refining node signatures.

Signature propagation is performed first in the forward direction and this is iterated while equivalent classes of signatures are changing. It is important to note that node labels are assigned as soon as they are uniquely identified. If non-singleton nodes exist after the forward iteration, then propagation is done in the backward direction starting with the already computed node signatures.

The equivalence classes of nodes, in terms of their signatures, either define isomorphic sub-graphs, or present rare hard cases calling for more elaborate refinement strategies discussed next. If all nodes have been assigned canonical labels at this point, we conjecture that the reordering provides a canonical mapping.

The computation of node signatures as sums of edge values is motivated by the need to distinguish nodes based on their structural information, such as the level-by-level distribution of nodes and complemented edges in the transitive fanin/fanout cones of the node. 


\subsection{Breaking ties}

As an example of the need for tie-breaking, consider first the left branch of the AIG shown in Figure 3.5. The transitive fanin cone of node $\mathrm{F}$ is composed of two isomorphic groups of AIG nodes (representing XOR gates). Thus the fanins of $F$ would not have unique node labels after signature refining.

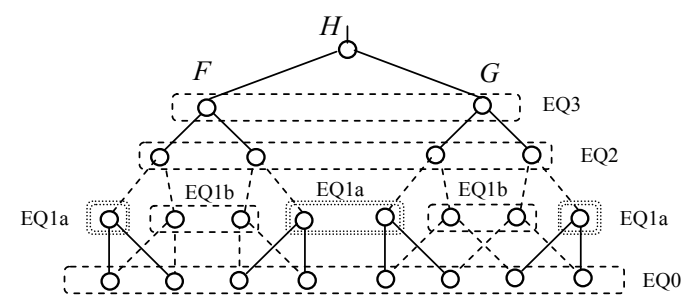

Figure 3.5: Illustration of node equivalence classes.

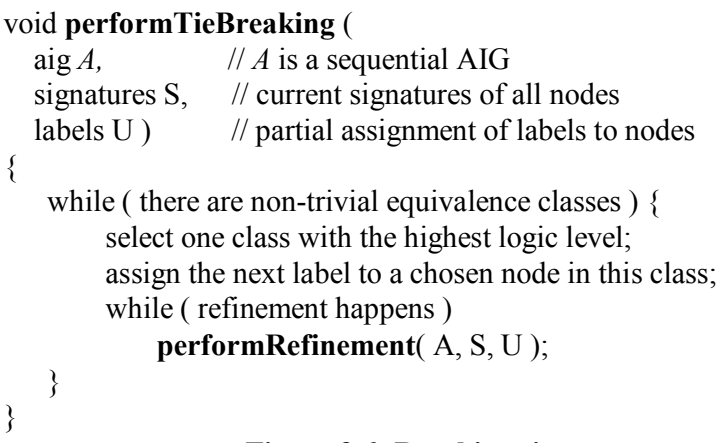

Figure 3.6: Breaking ties.

However, this can happen also even if the graph has does not have isomorphic sub-graphs. Consider the transitive fanin cone of node $\mathrm{H}$ in Figure 3.5 (ignoring the fact that $\mathrm{F}$ contains isomorphic sub-graphs). The equivalence classes of nodes in this cone are shown in dotted rectangles. In particular, equivalence class EQ3, composed of structurally different nodes $\mathrm{F}$ and $\mathrm{G}$, cannot be refined by forward and backward signature propagation. This is because transitive fanin/fanout cones of nodes $F$ and $G$ have the same levelby-level distribution of nodes and complemented edges.

If there are unlabeled nodes after signature propagation, we do a heuristic refinement by selecting one equivalence class and assigning its representative the next available label, as shown in Figure 3.6. This effect is propagated to distinguish other nodes. This refinement is continued until there are no unassigned nodes.

\subsection{Main procedure}

The top-level algorithm is shown in Figure 3.7.

\subsection{Creating the semi-canonical AIGER file and checking isomorphism}

Once the semi-canonical labels have been computed, we sort the nodes in the order of their new labels along with their fanins ordered according to increasing fanin node label (with inverted edges having their source node id negated). This produces a sequence of $N$ triples which can be written into an AIGER file [2]. The check for isomorphism is done simply by traversing the semicanonical triplets in order for the two files and comparing triplets. If ever there is a mismatch, the two AIGs are declared non-isomorphic, otherwise they are proved isomorphic because the node labeling provides an isomorphic one-to-one mapping between the two graphs.

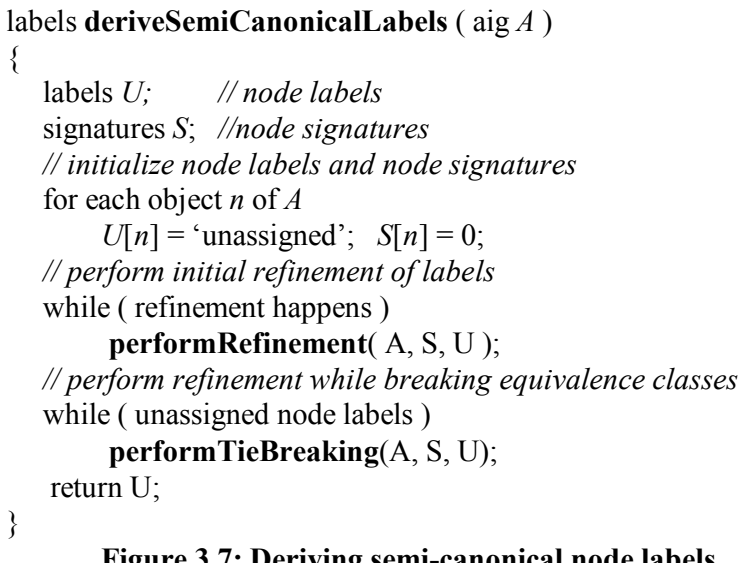

\subsection{Filtering isomorphic primary outputs}

We can detect and remove POs whose sequential logic cones are proved isomorphic to sequential logic cones of other POs in the AIG. The method is shown in Figure 3.8.

Computation in Figure 3.8 begins by detecting an overapproximation of equivalence classes of primary outputs using procedure computeApproxPoEquivClasses(). This procedure applies only forward signature propagation, which corresponds to quitting after the first while-loop in procedure performRefinement() shown in Figure 3.4.

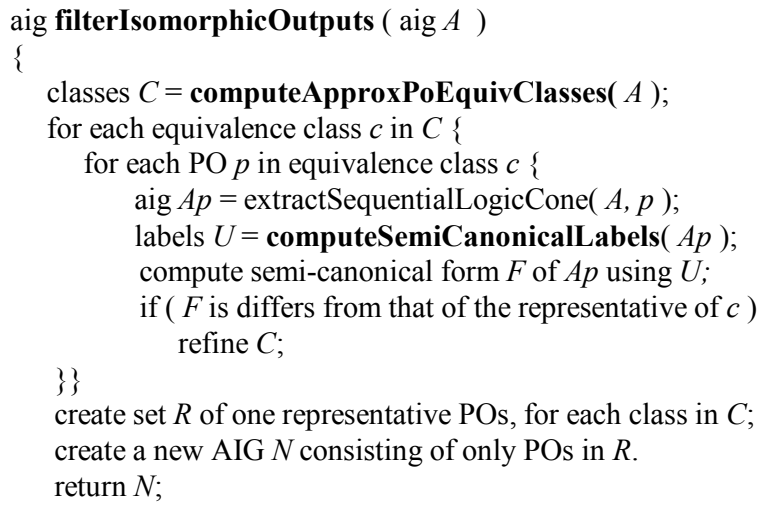

Figure 3.8: Filtering structurally isomorphic POs.

As a result, only the POs with different signatures of their transitive fanin cones are distinguished. For example, Figure 3.9 shows three structurally different AIGs. The first two can be distinguished by the forward procedure, because one of them has a complemented edge (dashed) while the other does not. The last two cannot be distinguished because the node signatures after forward propagation are identical in this case, so backward propagation is needed. 


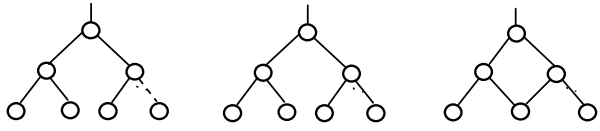

Figure 3.9: Three structurally different AIGs.

After this, if there are non-singular classes, these are checked for isomorphism and, if conflicts are found, these are refined. At the end, the remaining POs are truly the representatives of isomorphic equivalence classes.

\section{Experimental results}

\subsection{Public benchmarks}

The procedure for filtering isomorphic primary outputs, shown in Figure 3.7, is implemented as command \&iso in $\mathrm{ABC}$ [3][4]. It takes a sequential AIG and removes the POs whose sequential logic cones are isomorphic to those of some other PO (i.e. keeps only the class representatives).

Command \&iso can be used, for example, in property verification when the problem is a multi-output sequential miter. Applying \&iso reduces the miter to include only an irredundant set of properties. If a property is proved UNSAT, all the properties belonging to the same class are UNSAT. If a counter-example is derived, it can be mapped into counter-examples for all properties in the same class.

Results for the largest 8 circuits from the ISCAS benchmark suite are shown in Table 4.1.

The first column shows the benchmark name. The second column shows the number of AND nodes in the AIG. The next three columns compare the number of PO equivalence classes in (a) the original circuit, (b) after partial refinement with procedure computeApproxPoEquivClasses performing only forward signature propagation and (c) after complete refinement by \&iso. Finally, the last two columns show the runtime of forward signature propagation and that of \&iso. This experiment was performed using IntelCore 2Quad Q9450, 2.66GHz. Only one core was used.

Observations from Table 4.1:

1. There are many isomorphic outputs.

2. Runtimes are small (2 sec for all benchmarks).

3. Approximate classes are close to the real isomorphic classes.

\subsection{Industrial benchmarks}

In the second experiment, shown in Table 4.2, command \&iso was applied to a suite of 19 multi-output industrial verification benchmarks. Each benchmark was converted into a set of single-output problems using $\mathrm{ABC}$ command sequence "cone $-S-O<$ num $>$; scl" and given to ' $p d r$ ' [11], the most powerful single-engine prover among those currently available in ABC.

The first column of Table 4.2 shows the number of a verification problem. The following first section of the table shows benchmark statistics before applying \&iso while the second section shows statistics after applying \&iso. Inside each section, columns labeled "AND", "FF", "PI", and
"PO" show the number AND nodes, flip-flops, primary inputs and primary outputs. The columns labeled "pdr" show the number of POs solved by 'pdr' within the resource limits (5 minutes and 1GB). Columns labeled "time" show the cumulative runtime of 'pdr' on those POs that were successfully solved. Finally, the last column shows the runtime of \&iso for each benchmark. The bottom row shows geometric averages of the corresponding columns.

This experiment was performed on a cluster of machines running Linux 2.6 on a $2.4 \mathrm{GHz}$ AMD Opteron processor.

Observations from Table 4.2:

1. The number of POs is reduced almost $3 \mathrm{x}$.

2. The number of ANDs and flops is reduced $40 \%$.

3. The total verification time is reduced about $2 \mathrm{x}$, while \&iso takes only $15 \%$ of the total runtime.

\subsection{Measuring the precision of \&iso}

The algorithm implemented in command \&iso cannot be perfect, because is relies on tie-breaking to resolve ambiguities randomly, but choosing nodes in a given order.

Initial Monte Carlo experiments showed that in many cases \&iso finds all isomorphisms but we found a few where the hit rate was only about $7 \%-14 \%$.

The Monte Carlo experiments were done by choosing a non-trivial single-output cone from one of the 21 industrial examples of Table 4.2. This was written out as filel.aig During this writing, the $-u$ option causes the AIG to be written in a canonical order. Next, the original cone is permuted using $\mathrm{ABC}$ command permute. This randomly reorders the PIs, POs and FFs of the AIG. Then, it is written using the $-u$ option as file2.aig. Finally file1 and file 2 are compared for being identical using diff. This was repeated 100 times, recording when the two files were the same.

Although the results for some single-output cones are disappointing, precision is $100 \%$ on many benchmarks.

We also experimented on multi-output benchmarks by applying permute followed by \&iso and comparing the number of isomorphic POs before and after permutation. The numbers always matched. We conjecture that this discrepancy is because in a multi-output benchmark, the PIs and FFs are permuted in the same way for all POs. So when each cone is extracted, the set of output cones are "entangled" and isomorphism detection is more precise.

\section{Conclusions}

Related previous work includes methods for symmetry detection, focused on computing automorphisms of digital circuits [5][6][9][13][18] and functional symmetries [16][19] with applications in BDD variable reordering [17], reachability analysis [8], logic synthesis [15], SAT [1][12], and post-placement rewiring [7], to mention just a few.

We believe this is the first paper to propose an algorithm for semi-canonical labeling [14] of sequential AIGs. This method might be extended to general directed graphs, where a feedback node cut set plays the role of flip-flops. If the cut set is identified uniquely, then the method of this paper can be applied to identify isomorphisms. 
The proposed method and its public implementation in $\mathrm{ABC}$ can be used to avoid repeated time-consuming computations in industrial synthesis and verification tools.

\section{Acknowledgements}

This work is partly supported by SRC contract 1875.001 and NSA grant "Enhanced equivalence checking in cryptoanalytic applications". We also thank industrial sponsors of BVSRC: Altera, Atrenta, Cadence, Calypto, IBM, Intel, Jasper, Mentor Graphics, Microsemi, Real Intent, Synopsys, Tabula, and Verific for their continued support.

\section{References}

[1] F. A. Aloul, A. Ramani, I. L. Markov, and K. A. Sakallah, "Solving difficult SAT instances in the presence of symmetry", IEEE TCAD'03, vol. 22(9), pp. 1117-1137.

[2] A. Biere, AIGER format. http://fmv.jku.at/aiger/

[3] Berkeley Logic Synthesis and Verification Group.. http://wwwcad.eecs.berkeley.edu/ alanmi/abc

[4] R. Brayton and A. Mishchenko, "ABC: An academic industrialstrength verification tool", Proc. CAV'10, LNCS 6174, pp. 24-40.

[5] D. Chai and A. Kuehlmann, "A compositional approach to symmetry detection in circuits", Proc. IWLS'06, pp. 228-234.

[6] D. Chai and A. Kuehlmann, "Symmetry detection for large multioutput functions", Proc. IWLS'07, pp. 305-311.

[7] K.-H. Chang, I. L. Markov and V. Bertacco, "Post-placement rewiring by exhaustive search for functional symmetries", $A C M$ TODAES, vol. 12(3), \#32, August 2007.
[8] P. Chauhan, P. Dasgupta, and P. P. Chakraborty, "Exploiting graph isomorphism for BDD compaction and faster simulation", Proc. VLSI'99, pp. 224-229.

[9] P. T. Darga, K. A. Sakallah, and I. L. Markov. "Faster symmetry discovery using sparsity of symmetries". Proc.DAC'08, pp.149-154.

[10] N. Een and A. Biere, "Effective preprocessing in SAT through variable and clause elimination", Proc. SAT'05.

[11] N. Een, A. Mishchenko and R. Brayton, "Efficient implementation of property-directed reachability", Proc. FMCAD'11.

[12] H. Katebi, K. A. Sakallah, and I. L. Markov, "Symmetry and satisfiability: An update", Proc. SAT'10, pp. 113-127.

[13] H. Katebi, K. A. Sakallah, and I. L. Markov, "Conflict anticipation in the search for graph automorphisms", Proc. Int'l Conf. on Logic for Programming, Artificial Intelligence and Reasoning (LPAR), Merida, Venezuela, 2012.

[14] B. D. McKay, "Computing automorphisms and canonical labeling of graphs", Combinatorial Mathematics, Lecture Notes in Mathematics, 686, pp. 223-232 (Springer-Verlag, Berlin, 1978).

[15] V. N. Kravets and K. A. Sakallah, "Constructive library-aware synthesis using symmetries". Proc. DATE'00, pp. 208-213.

[16] A. Mishchenko, "Fast computation of symmetries in Boolean functions", IEEE TCAD'03, vol. 22(11), pp.1588-1593.

[17] S. Panda, F. Somenzi, and B. Plessier, "Symmetry detection and dynamic variable ordering of decision diagrams". Proc. ICCAD'94.

[18] G. Wang, A. Kuehlmann, and A. Sangiovanni-Vincentelli, "Structural detection of symmetries in Boolean functions", Proc. ICCD'03, pp. 498-503.

[19] J. S. Zhang, A. Mishchenko, R. Brayton, and M. Chrzanowska-Jeske, "Symmetry detection for large boolean functions using circuit representation, simulation, and satisfiability", $D A C^{\prime}$ '06, 510-515.

Table 4.1: Computing canonical structure for largest ISCAS benchmarks.

\begin{tabular}{|l|r|r|r|r|r|r|}
\hline \multicolumn{1}{|c|}{ Example } & \multicolumn{1}{|c|}{ AIG } & \multicolumn{1}{c|}{ POs } & \multicolumn{1}{c|}{ POs filter } & \multicolumn{1}{c|}{ POs canon } & \multicolumn{1}{c|}{ Time filter } & Time canon \\
\hline s13207 & 2728 & 121 & 88 & 89 & 0.01 & 0.11 \\
\hline s13207_1 & 2728 & 152 & 89 & 89 & 0.01 & 0.05 \\
\hline s15850 & 3526 & 87 & 43 & 43 & 0.01 & 0.05 \\
\hline s15850_1 & 3526 & 150 & 47 & 32 & 0.01 & 0.01 \\
\hline s35932 & 11948 & 320 & 320 & 39 & 0.01 & 0.02 \\
\hline s38417 & 9238 & 106 & 218 & 0.01 & 0.53 \\
\hline s38584 & 12310 & 278 & 218 & & 0.01 & 0.53 \\
\hline s38584_1 & 12310 & 304 & 219 & & & 0.01 \\
\hline
\end{tabular}

Table 4.2: Reductions on industrial verification benchmarks and impact on cumulative verification runtime.

\begin{tabular}{|c|c|c|c|c|c|c|c|c|c|c|c|c|c|}
\hline & \multicolumn{6}{|c|}{ Industrial circuits in their original form } & \multicolumn{6}{|c|}{ Industrial circuits after \&iso is applied } & \&iso \\
\hline Ex & AND & $\mathrm{FF}$ & PI & $\mathrm{PO}$ & PDR & time, $\mathrm{s}$ & AND & $\mathrm{FF}$ & PI & $\mathrm{PO}$ & PDR & time, $\mathrm{s}$ & time, $\mathrm{s}$ \\
\hline 0 & 3307 & 64 & 54 & 40 & 40 & 38.8 & 3177 & 64 & 54 & 20 & 20 & 36.8 & 0.01 \\
\hline 1 & 25478 & 184 & 138 & 184 & 136 & 15.8 & 19493 & 65 & 138 & 65 & 17 & 1.9 & 0.05 \\
\hline 2 & 248642 & 1291 & 767 & 31 & 0 & 0.1 & 168090 & 951 & 767 & 21 & 0 & 0.1 & 1.76 \\
\hline 3 & 1037 & 56 & 5 & 18 & 0 & 0.1 & 970 & 52 & 5 & 9 & 0 & 0.1 & 0.02 \\
\hline 4 & 217292 & 2483 & 2277 & 1203 & 34 & 3.4 & 217202 & 2483 & 2277 & 1188 & 34 & 3.4 & 215.71 \\
\hline 5 & 58432 & 6 & 3464 & 2928 & 2928 & 661.9 & 36745 & 6 & 3464 & 1654 & 1654 & 373.0 & 19.46 \\
\hline 6 & 85926 & 4156 & 6510 & 907 & 907 & 90.7 & 85926 & 4156 & 6510 & 904 & 904 & 90.4 & 15.23 \\
\hline 7 & 162357 & 331 & 1019 & 24 & 12 & 1.2 & 162269 & 331 & 1019 & 13 & 1 & 0.1 & 0.07 \\
\hline 8 & 217292 & 2483 & 2277 & 1203 & 34 & 3.8 & 217202 & 2483 & 2277 & 1188 & 34 & 3.8 & 738.76 \\
\hline 9 & 259731 & 10993 & 2362 & 181 & 73 & 3430.2 & 255978 & 10859 & 2362 & 164 & 64 & 3429.3 & 7.05 \\
\hline 10 & 22325 & 1289 & 492 & 165 & 165 & 16.5 & 11650 & 596 & 492 & 60 & 60 & 6.0 & 0.14 \\
\hline 11 & 180873 & 7129 & 14046 & 12938 & 0 & 0.1 & 92358 & 2791 & 14046 & 4702 & 0 & 0.1 & 28.70 \\
\hline 12 & 94912 & 8298 & 4210 & 32 & 32 & 748.9 & 3307 & 269 & 4210 & 1 & 1 & 20.6 & 0.15 \\
\hline 13 & 212389 & 229 & 1363 & 128 & 118 & 95.5 & 211514 & 229 & 1363 & 27 & 17 & 81.8 & 0.60 \\
\hline 14 & 1009803 & 66962 & 125 & 28 & 0 & 0.1 & 135545 & 8624 & 125 & 21 & 0 & 0.1 & 1.41 \\
\hline 15 & 74480 & 2976 & 394 & 327 & 0 & 0.1 & 18765 & 900 & 394 & 14 & 0 & 0.1 & 4.16 \\
\hline 16 & 63855 & 6806 & 1519 & 413 & 405 & 226.9 & 63149 & 6805 & 1519 & 60 & 52 & 161.2 & 0.40 \\
\hline 17 & 10130 & 64 & 33 & 16 & 2 & 0.2 & 9948 & 64 & 33 & 6 & 1 & 0.1 & 0.05 \\
\hline 18 & 162357 & 331 & 1019 & 24 & 12 & 2.1 & 162269 & 331 & 1019 & 13 & 1 & 0.2 & 0.11 \\
\hline $\mathrm{Geo}$ & 1.000 & 1.000 & 1.000 & 1.000 & 1.000 & 1.000 & 0.611 & 0.597 & 1.000 & 0.385 & 0.396 & 0.495 & 0.161 \\
\hline
\end{tabular}

